\title{
Comparison of stabilizing mechanisms between discrete- and continuous-time consumer-resource models
}

\author{
Abhyudai Singh ${ }^{1}$
}

\begin{abstract}
There is rich literature on using continuous-time and discrete-time models for studying population dynamics of consumer-resource interactions. The continuous-time framework is generally used to model populations with overlapping generations and all year-round reproduction. In contrast, discrete-time models are more suited for populations with non-overlapping generations that reproduce in a discrete pulse determined by season. Inspired by the Nicholson-Bailey/Lotka-Volterra modeling formalisms in discrete-time/continuous-time, respectively, we consider host-parasitoid interactions with an arbitrary parasitoid attack rate that is a function of both the host and parasitoid population densities. We characterize and compare stability regimes in both modeling frameworks for analogous host reproduction and attack rates. Our analysis shows that a Type II functional response is stabilizing in both modeling frameworks only when combined with other mechanisms, such as mutual interference between parasitoids. This stability regime related to a Type II functional response is smaller in the discrete-time framework compared to continuous-time, and shrinks with increasing host reproduction. A Type III functional response is by itself stabilizing, but the extent of attack-rate acceleration needed is much higher in the discrete-time framework, and its stability regime expands with increasing host reproduction. Finally, our results show that while mutual parasitoid interference can stabilize population dynamics, cooperation between parasitoids to handle hosts is destabilizing in both frameworks. However, a combination of a Type III functional response together with parasitoid cooperation can create stability. In summary, our comparative analysis systematically characterizes diverse ecological processes driving stable population dynamics in discrete-time and continuous-time consumer-resource models.
\end{abstract}

\section{INTRODUCTION}

Interaction between a resource (such as, a prey or host) and a consumer (such as, a predator or parasitoid) forms a core motif in ecological networks. Population dynamics of consumer-resource interactions has been extensively studied using two different approaches: continuous-time and discrete-time models. Perhaps, the classical example of this in continuous-time is the Lotka-Volterra model

$$
\begin{aligned}
\frac{d h(t)}{d t} & =r h(t)-\operatorname{ch}(t) p(t) \\
\frac{d p(t)}{d t} & =\operatorname{ch}(t) p(t)-\gamma p(t)
\end{aligned}
$$

[1]-[8]. Here $h(t)$ and $p(t)$ denote the population densities of the host and the parasitoid at time $t$, respectively. In the absence of the consumer, host density increases exponentially over time with growth rate $r$. Parasitoids attack hosts with rate $c$ with each parasitized host developing into a new parasitoid. Finally, $1 / \gamma$ is the average lifespan of an individual parasitoid. It is well-known that the steady-state equilibrium of the Lotka-Volterra model is neutrally stable resulting in cycling population densities with a period of $\approx 2 \pi / \sqrt{r \gamma}[9]$.

The analogous counterpart of the Lotka-Volterra model in discrete-time is the Nicholson-Bailey model

$$
\begin{aligned}
H_{t+1} & =R H_{t} \exp \left(-c P_{t}\right) \\
P_{t+1} & =R H_{t}\left[1-\exp \left(-c P_{t}\right)\right]
\end{aligned}
$$

where we now use capital letters $H_{t}$ and $P_{t}$ to denote the adult host, and the adult parasitoid densities, respectively, in year $t$ [3], [10]-[13]. The model reflects the synchronized annual life cycles of these insects living in the temperate regions of the world [14]-[17]. More specifically, if $R>1$ denotes the number of viable eggs produced per adult host, then $R H_{t}$ is the host larval density that becomes exposed to parasitoid attacks. Parasitoids attack and parasitize hosts at a constant rate $c$, resulting in the fraction $\exp \left(-c P_{t}\right)$ that escapes parasitism to become the adult hosts for

\footnotetext{
${ }^{1}$ Abhyudai Singh is with the Departments of Electrical and Computer Engineering, Biomedical Engineering and Mathematical Sciences, University of Delaware, Newark, DE 19716, USA absingh@udel.edu
} 
the next year. Similarly, the fraction $1-\exp \left(-c P_{t}\right)$ of parasitized larvae give rise to adult parasitoids in the next generation. The Nicholson-Bailey model is characterized by diverging oscillations in population densities resulting in an unstable population dynamics [10], and several studies have characterized ecological factors promoting stability in host-parasitoid interactions, especially in the context of parasitoid-mediated biological control of pest species [9], [18]-[21].

In this contribution, we consider generalized Lotka-Volterra and Nicholson-Bailey models with a densitydependent parasitoid attack rate, and systematically compare the stability regions for a combination of ecology processes (functional responses, mutual cooperation or interference between parasitoids, etc.) The manuscript is organized as follows: in Section II we formulate the generalized Lotka-Volterra model and derive criterion for stable population dynamics. The same process is repeated in Section III for an analogously formulated NicholsonBailey model. We compare stability regions in Section IV putting results in the context of known literature and highlighting new insights arising from this study.

\section{STABILITY CONDITION FOR A GENERALIZED LOTKA-VOLTERRA MODEL}

Consider a generalized Lotka-Volterra model

$$
\begin{aligned}
\frac{d h(t)}{d t} & =r h(t)-f(h, p) h(t) p(t) \\
\frac{d p(t)}{d t} & =f(h, p) h(t) p(t)-\gamma p(t)
\end{aligned}
$$

with an attack rate $f(h, p)$ that is an arbitrary continuously-differentiable function of both the host and parasitoid population densities. Such an attack rate captures diverse ecological processes:

- Function $f$ decreasing (increasing) with host density corresponds to a Type II (Type III) functional response [22]-[31].

- Function $f$ decreasing (increasing) with parasitoid density corresponds to mutual interference (cooperation) between parasitoids [32]-[36].

We assume that model (3) has a unique equilibrium given as the solution to

$$
f\left(h^{*}, p^{*}\right)=\frac{r}{p^{*}}, \quad p^{*}=\frac{r h^{*}}{\gamma} .
$$

where $h^{*}$ and $p^{*}$ represent the equilibrium host and parasitoid densities, respectively. Our analysis in Appendix A shows that this equilibrium is asymptotically stable, if and only if,

$$
f_{p}<\frac{r f_{h}}{\gamma}, 1+f_{h}+f_{p}>0
$$

where $f_{p}$ and $f_{h}$

$$
\begin{aligned}
f_{h} & :=\left.\frac{h^{*}}{f\left(h^{*}, p^{*}\right)} \frac{\partial f(h, p)}{\partial h}\right|_{h=h^{*}, p=p^{*}}, \\
f_{p} & :=\left.\frac{p^{*}}{f\left(h^{*}, p^{*}\right)} \frac{\partial f(h, p)}{\partial p}\right|_{h=h^{*}, p=p^{*}},
\end{aligned}
$$

are the dimensionless log sensitivities of the attack rate to the host and parasitoid densities, respectively. It is reasonable to have the net attack rate per host $f(h, p) p$ be a non-decreasing function of the parasitoid density that constrains $f_{p} \geq-1$. Similarly, we constrain $f_{h} \geq-1$ that ensures the net attack rate per parasitoid $f(h, p) h$ be a non-decreasing function of the host density. If any one of the inequalities in (5) does not hold, then the equilibrium is locally unstable and this instability often manifests in a stable limit cycle [37], [38]. 


\section{StABILITY CONDITIONS FOR A GENERALIZED NiCHOLSON-BAILEY MODEL}

We next consider the discrete-time counterpart of the generalized Lotka-Volterra model. One phenomenological approach to obtain this model is to simply substitute $c$ in (2) with $f\left(H_{t}, P_{t}\right)$ yielding

$$
\begin{aligned}
H_{t+1} & =R H_{t} \exp \left(-f\left(H_{t}, P_{t}\right) P_{t}\right) \\
P_{t+1} & =R H_{t}\left[1-\exp \left(-f\left(H_{t}, P_{t}\right) P_{t}\right)\right]
\end{aligned}
$$

[39]. Note that during the time window when hosts are vulnerable to parasitoid attacks, the density of unparasitized host continuously decreases. To capture such effects of continuously changing populations, a semi-discrete or hybrid formalism has been proposed to mechanistically formulate the corresponding discrete-time model [40]-[46]. Briefly, an ordinary-differential equation model describing population interaction during the attack time window is solved to derive the update functions of the discrete-time model. Importantly, this mechanistic approach can yield contrasting results from the phenomenological approach - a Type III functional response is stabilizing in the semi-discrete approach [43], [47], but always destabilizing if one considers (8) with an analogous attack rate [43].

Referring the reader to Appendix B for details, the semi-discrete approach yields the following model in the discrete-time formalism that is analogous to the continuous-time counterpart (3)

$$
\begin{aligned}
& H_{t+1}=\frac{R H_{t}}{\left(1+f_{h}\left(R H_{t}\right)^{f_{h}} P_{t}^{1+f_{p}}\right)^{\frac{1}{f_{h}}}} \\
& P_{t+1}=R H_{t}\left(1-\frac{1}{\left(1+f_{h}\left(R H_{t}\right)^{f_{h}} P_{t}^{1+f_{p}}\right)^{\frac{1}{f_{h}}}}\right) .
\end{aligned}
$$

As before, $f_{h}$ and $f_{p}$ are the $\log$ sensitivities of the parasitoid attack rate to the host and parasitoid densities, respectively. This model as a unique non-trivial equilibrium

$$
H^{*}=\left(\frac{1-R^{-f_{h}}}{f_{h}(R-1)^{1+f_{p}}}\right)^{\frac{1}{1+f_{h}+f_{p}}}, \quad P^{*}=(R-1) H^{*},
$$

which is asymptotically stable, if and only if.

$$
f_{p}<\frac{R+\left(f_{h}(R-1)-R\right) R^{f_{h}}}{R\left(R^{f_{h}}-1\right)}, 1+f_{h}+f_{p}>0 .
$$

Note that the second inequality is the same as in (5), and hence $1+f_{h}+f_{p}>0$ is a necessary condition for stability in both modeling frameworks. One can consider small and larger values of $R$ for which the stability condition reduces to

$$
\begin{aligned}
& f_{p}<0, \quad 1+f_{h}+f_{p}>0, \quad \text { when } \quad R \rightarrow 1, \\
& f_{p}<f_{h}-1, \quad 1+f_{h}+f_{p}>0, \quad \text { when } \quad R \rightarrow \infty,
\end{aligned}
$$

respectively.

\section{DISCUSSION}

To understand the stabilizing/destabilizing effects of diverse ecological processes we plot the stability regions as predicted by inequalities (5) and (11) in Fig. 1. For an analogous comparison, the hosts growth rate $r$ in the continuous-time framework is related to $R$ in the discrete-time framework by $r=\log (R)$. In Fig. $1, f_{h}=f_{p}=0$ represents the classical Lotka-Volterra/Nicholson-Bailey models and

- Going right along the positive $\mathrm{x}$-axis $\left(f_{h}>0\right)$ corresponds to an attack rate that accelerates with increasing host density as in a Type III functional response.

- Going left along the negative $\mathrm{x}$-axis $\left(f_{h}<0\right)$ corresponds to an attack rate that decreases with increasing host density as in a Type II functional response. 
Discrete-time framework
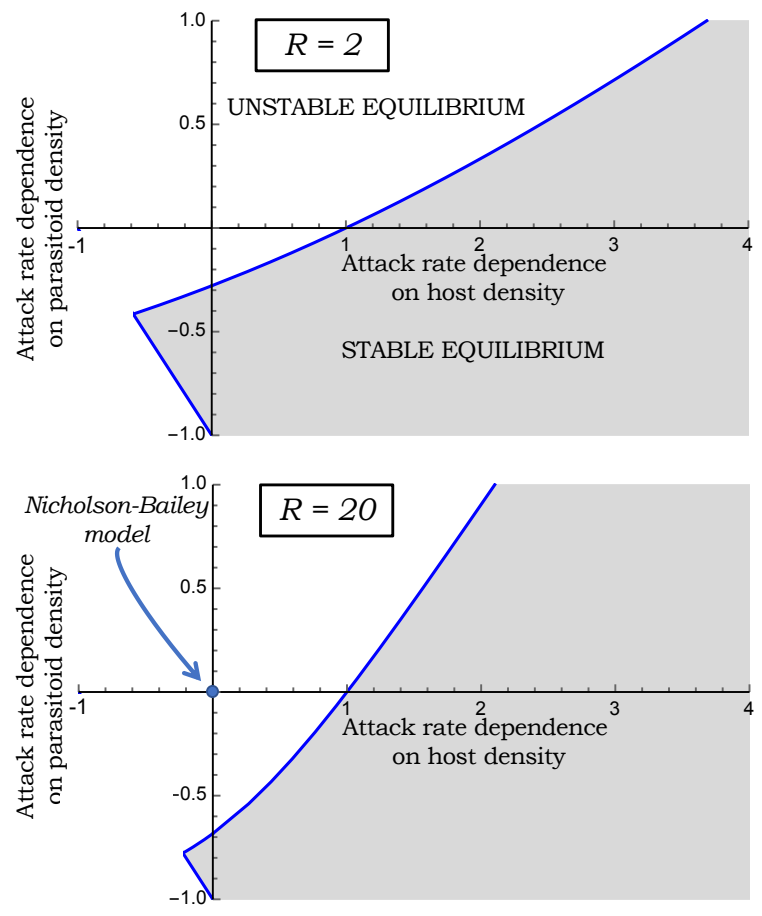
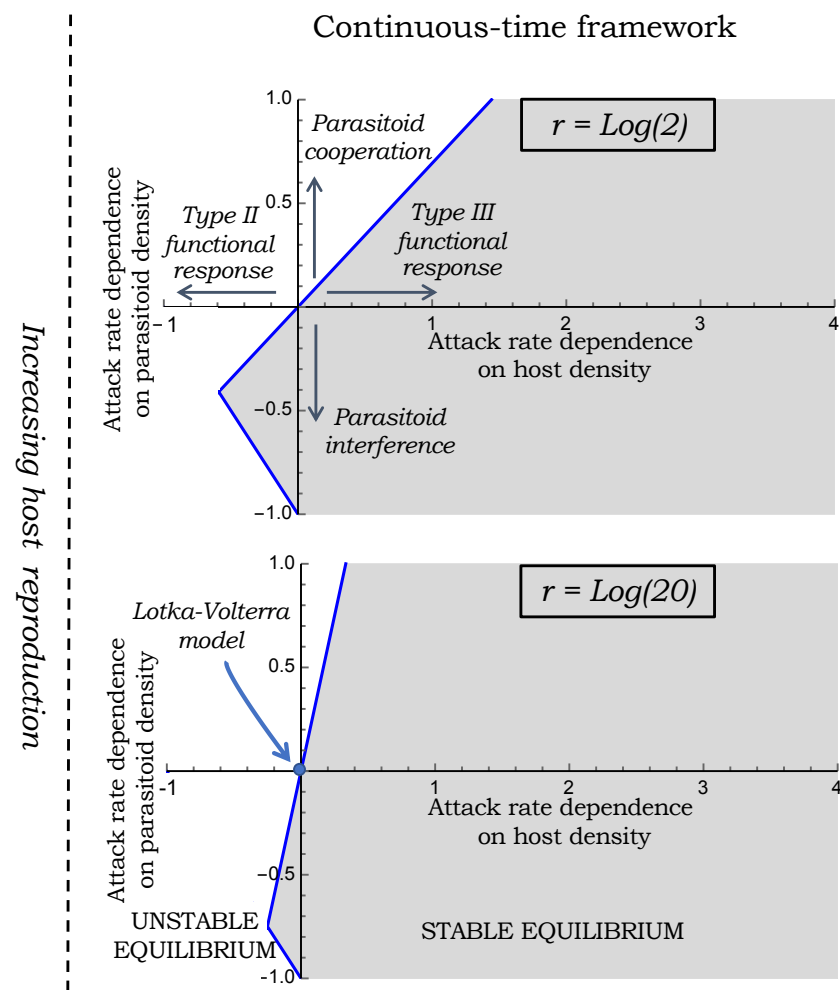

Fig. 1: The stability region (grey shaded area) as determined by (5) and (11) in continuous-time (right) and discretetime (left), respectively, is plotted as function of $f_{h}$ (attack-rate sensitivity to the host density) and $f_{p}$ (attack-rate sensitivity to the parasitoid density). The origin $f_{h}=f_{p}=0$ represents the classical Lotka-Volterra and NicholsonBailey models. While the former model in continuous time is on the edge of stabliity indicating a neutrally-stable equilibrium, the Nicholson-Bailey model in discrete time is outside the grey shaded area indicating an unstable equilibrium. The stability regions are plotted for two different levels of host reproduction $-R=2$ (top) and $R=20$ (bottom) with $r=\log R$ and $\gamma=1$.

- Going up along the positive y-axis $\left(f_{p}>0\right)$ corresponds to an attack rate that increases with parasitoid density capturing cooperation between parasitoids to handle hosts.

- Going down along the negative $\mathrm{y}$-axis $\left(f_{p}<0\right)$ corresponds to an attack rate that decreases with increasing parasitoid density corresponding to mutual interference between parasitoid, or aggregation of parasitoid attacks to a subpopulation of high-risk hosts [19], [43], [48]-[55].

A straightforward observation from Fig. 1 is that the region of stability is larger in continuous time as compared with discrete time, but in some limits they turn out to be identical.

\section{A. Comparison of stability conditions with respect to host reproduction}

An important result that emerges from the inequalities (5) and (11) is that for low levels of host reproduction (i.e., $R \rightarrow 1$ and $r \rightarrow 0$ ), both modeling frameworks have the exact same stability criterion

$$
f_{p}<0, \quad 1+f_{h}+f_{p}>0
$$

showing that an attack rate decreasing with increasing parasitoid density is a necessary condition for stability. For high levels of host reproduction (i.e., $R \rightarrow \infty$ and $r \rightarrow \infty$ ), $f_{h}>0$ becomes the necessary and sufficient condition for stability in the Lotka-Volterra framework. In the Nicholson-Bailey framework, the stability condition for $R \rightarrow \infty$ is given by (12b) that corresponds to the two lines meeting at $f_{h}=0$ and $f_{p}=-1$. This implies that $f_{h}>0$ is only 
a necessary condition for stability in the discrete-time framework for large values of $R$ and one further requires $f_{p}<f_{h}-1$ for stability.

\section{B. Impact of functional responses}

Consistent with [56], our analysis shows that a Type II functional response with $f_{h}<0$ and $f_{p}=0$ will lead to an unstable equilibrium in both frameworks. Interestingly, a Type II functional response $\left(f_{h}<0\right)$ can provide stability if combined with other mechanisms where $f_{p}<0$ (grey-shaded area in the third quadrant). The meeting point of two lines in the third quadrant gives the necessary condition for stability

$$
\begin{aligned}
& f_{h}>-\frac{1}{1+\frac{r}{\gamma}} \quad \text { in continuous time } \\
& f_{h}>\frac{\log R-\log (2 R-1)}{\log R} \quad \text { in discrete time }
\end{aligned}
$$

that reduces to $f_{h}>-1$ in the limit $R \rightarrow 1 \& r \rightarrow 0$. A Type III functional response $\left(f_{h}>0\right.$ and $\left.f_{p}=0\right)$ is stabilizing in both framework but the degree of attack-rate acceleration needed for stability is much higher in the discrete-time framework: $f_{h}>1$ in the discrete-time framework compared to $f_{h}>0$ in the continuous-time framework.

Recall that one of the inequalities needed for stability $1+f_{h}+f_{p}>0$ is the same for both frameworks. The other inequality in the continuous-time framework is anchored at the origin and rotates anticlockwise with increasing $R$. In contrast, the other inequality in the discrete-time framework is anchored at $f_{h}=1, f_{h}=0$ and also rotates anticlockwise with increasing $R$. This leads to the stability region related to a Type II functional response shrinking with increasing $R$. However, the stability region related to Type III responses expands with increasing $R$ in the continuous-time framework. The non-origin anchoring in the discrete-time framework leads to the stability region related to Type III responses expanding with increasing $R$ for $f_{h}>1$, but shrinking for $0<f_{h}<1$.

\section{Impact of a parasitoid-dependent attack rate}

For a parasitoid-dependent attack rate $\left(f_{h}=0\right)$ one can see that $f_{p}>0$ (i.e., cooperation between parasitoids) is not stabilizing in both frameworks. In contrast, mutual interference between parasitoids is stabilizing with the stability criterion reducing to

$$
\begin{array}{r}
f_{p}<0 \quad \text { in continuous time } \\
f_{p}<-\frac{R \ln (R)+1-R}{R \ln (R)} \quad \text { in discrete time. }
\end{array}
$$

An interesting observation from the fourth-quadrant of the discrete-time stability region is that while value of

$$
0>f_{p}>-\frac{R \ln (R)+1-R}{R \ln (R)}
$$

and

$$
0<f_{h}<1
$$

are by themselves not stabilizing, their combination can lead to stability. Thus, moderate levels of parasitoid interference together with attack-rate acceleration to host density can stabilize population dynamics in the NicholsonBailey formulation of host-parasitoid population dynamics. Finally, we point out that the stability resulting from a combination of cooperation between parasitoids and a Type III functional response (grey-shaded region in the first quadrant), but increasing parasitoid cooperation also requires a higher degree of attack-rate acceleration to keep the system dynamics stable. 


\section{APPENDIX A: STABILITY CRITERION FOR THE GENERALIZED LOTKA-VOLTERRA MODEL}

Linearizing the right-hand-side of (3) around the equilibrium yields the following Jacobian matrix

$$
A=\left[\begin{array}{cc}
-r f_{h} & -f_{p} \gamma-\gamma \\
r f_{h}+r & \gamma f_{p}
\end{array}\right]
$$

and stability requires a Hurwitz matrix whose eigenvalues have negative real parts [57], [58]. For a two-dimensional system, the equilibrium is asymptotically stable, if and only if, the determinant of the $A$ matrix is positive and its trace is negative [57], [58]. This implies that the equilibrium obtained as the solution to (4) is asymptotically stable, if and only if, both inequalities in (5) hold.

\section{APPENDIX B: STABILITY CRITERION FOR THE GENERALIZED NICHOLSON-BAILEY MODEL}

The semi-discrete approach models the host-parasitoid interaction during the host's vulnerable stage as an ordinary differential equation. Let $\tau$ denote the time within the host vulnerable stage that varies from 0 to $T$ corresponding to the start and end of the vulnerable stage. The densities of parasitoids, un-parasitized and parasitized host larvae at time $\tau$ within the vulnerable stage of year $t$ are represented by $P(\tau, t), L(\tau, t), I(\tau, t)$, respectively. These densities evolve as per the dynamical system

$$
\begin{aligned}
& \frac{d P(\tau, t)}{d \tau}=-\gamma_{P} P(\tau, t) \\
& \frac{d L(\tau, t)}{d \tau}=-c P(\tau, t) L(\tau, t)-\gamma_{L} L(\tau, t) \\
& \frac{d I(\tau, t)}{d \tau}=c P(\tau, t) L(\tau, t)-\gamma_{I} I(\tau, t),
\end{aligned}
$$

where $c$ represents the parasitoid's attack rate per host, and $\gamma_{P}, \gamma_{L}, \gamma_{I}$ are the death rates of the respective species. Assuming $P_{t}$ parasitoids, $R H_{t}$ host larvae, and no parasitized larvae at the start of the vulnerable period $(\tau=0)$, solving the above differential equations with initial conditions

$$
L(0, t)=R H_{t}, \quad P(0, t)=P_{t}, \quad I(0, t)=0
$$

predicts the parasitized and unparasitized larval populations at the end of the season $(\tau=T)$. This leads to a more general discrete-time model

$$
\begin{aligned}
H_{t+1} & =F\left(H_{t}, P_{t}\right) \\
P_{t+1} & =G\left(H_{t}, P_{t}\right)
\end{aligned}
$$

where update functions are obtained by setting

$$
\begin{aligned}
& F\left(H_{t}, P_{t}\right)=L(T, t) \\
& G\left(H_{t}, P_{t}\right)=I(T, t) .
\end{aligned}
$$

Solving (19) for a constant attack rate $c$ with no mortalities $\left(\gamma_{P}=\gamma_{L}=\gamma_{I}=0\right)$, and assuming $T=1$ without loss of any generality, yields the Nicholson-Bailey model (2).

To capture a generalized parasitoid attack with $\log$ sensitivities $f_{h}$ (with respect to the host) and $f_{p}$ (with respect to the parasitoid), we replace $c$ in (19) with the monomial $L(\tau, t)^{f_{h}} P_{t}^{f_{p}}$. In the absence of any mortalities $\left(\gamma_{P}=\right.$ $\left.\gamma_{L}=\gamma_{I}=0\right)$ and again assuming $T=1$, the above semi-discrete approach results in the model (9). Linearizing the right-hand-side of (9) around the equilibrium (10) results in the Jacobian matrix

$$
A=\left[\begin{array}{lc}
1-f_{h} H^{* f_{h}} P^{* 1+f_{p}} & -\left(1+f_{p}\right) H^{* 1+f_{h}} P^{* f_{p}} \\
R-1+f_{h} H^{* f_{h}} P^{* 1+f_{p}} & \left(1+f_{p}\right) H^{* 1+f_{h}} P^{* f_{p}}
\end{array}\right],
$$

and stability in the discrete-time formalism requires all eigenvalues of $A$ to have an absolute value less than one [58], [59]. For a $2 \times 2$ matrix, the stability criterion can be written in terms of the determinant and the trace of $A$ 
- the equilibrium (10) is stable, if and only if,

$$
\begin{array}{r}
1-\operatorname{Det}(A)>0 \\
1+\operatorname{Tr}(A)-\operatorname{Det}(A)>0 \\
1+\operatorname{Tr}(A)+\operatorname{Det}(A)>0 .
\end{array}
$$

It turns out that the last inequality always holds, and the first two inequalities yield the stability conditions (11).

\section{REFERENCES}

[1] A. J. Lotka, Elements of physical biology. Williams \& Wilkins, 1925.

[2] V. Volterra, Variations and fluctuations in the number of individuals in cohabiting animal species. C. Ferrari, 1927.

[3] L. Edelstein-Keshet, Mathematical models in biology. SIAM, 2005.

[4] R. May, A. R. McLean, et al., Theoretical ecology: principles and applications. Oxford University Press on Demand, 2007.

[5] M. Begon and C. R. Townsend, Ecology: from individuals to ecosystems. John Wiley \& Sons, 2020.

[6] M. S. Bartlett, "On theoretical models for competitive and predatory biological systems," Biometrika, vol. 44, no. 1/2, pp. $27-42,1957$.

[7] R. Arditi and L. R. Ginzburg, How species interact: altering the standard view on trophic ecology. Oxford University Press, 2012.

[8] A. Singh, "Analytical discrete-time models of insect population dynamics," 2020.

[9] W. W. Murdoch, C. J. Briggs, and R. M. Nisbet, Consumer-Resouse Dynamics. Princeton,NJ: Princeton University Press, 2003.

[10] A. Nicholson and V. A. Bailey, "The balance of animal populations. part 1." Proc. of Zoological Society of London, vol. 3, pp. 551-598, 1935.

[11] R. M. May and M. P. Hassell, "The dynamics of multiparasitoid-host interactions," The American Naturalist, vol. 117, no. 3, pp. 234-261, 1981.

[12] E. Hackett-Jones, C. Cobbold, and A. White, "Coexistence of multiple parasitoids on a single host due to differences in parasitoid phenology,” Theoretical Ecology, vol. 2, no. 1, pp. 19-31, 2009.

[13] E. van Velzen, S. Pérez-Vila, and R. S. Etienne, "The role of within-host competition for coexistence in multiparasitoid-host systems," The American Naturalist, vol. 187, no. 1, pp. 48-59, 2016.

[14] A. E. Hajek, Insect parasitoids: attack by aliens. Cambridge University Press, 2004, p. 145169.

[15] H. C. J. Godfray, Parasitoids; Behavioral and Evolutionary Ecology. 41 William St, Princeton, NJ 08540: Princeton University Press, 1994.

[16] J. Waage and D. Greathead, Insect Parasitoids. Academic Press, 1986.

[17] M. E. Hochberg and A. R. Ives, Parasitoid population biology. Princeton University Press, 2000.

[18] M. P. Hassell. New York: Oxford University Press, 2000.

[19] A. Singh, W. W. Murdoch, and R. M. Nisbet, "Skewed attacks, stability, and host suppression," Ecology, vol. 90, no. 6, pp. 1679-1686, 2009.

[20] M. A. Jervis, B. A. Hawkin, and N. A. C. Kidd, "The usefulness of destructive host-feeding parasitoids in classical biological control: theory and observation conflict," Ecological Entomology, vol. 21, no. 1, pp. 41-46, 1996.

[21] N. Kakehashi, Y. Suzuki, and Y. Iwasa, "Niche overlap of parasitoids in host-parasitoid systems: its consequence to single versus multiple introduction controversy in biological control," Journal of Applied Ecology, pp. 115-131, 1984.

[22] D. J. Rogers, "Random searching and incest population models," J. of Animal Ecology, vol. 41, pp. 369-383, 1972.

[23] C. S. Holling, "The functional response of predators to prey density and its role in mimicry and population regulation," The Memoirs of the Entomological Society of Canada, vol. 97, no. S45, pp. 5-60, 1965

[24] F. Barraquand, "Functional responses and predator-prey models: a critique of ratio dependence," Theoretical ecology, vol. 7, no. 1, pp. $3-20,2014$.

[25] N. Mills and I. Lacan, "Ratio dependence in the functional response of insect parasitoids: evidence from trichogramma minutum foraging for eggs in small host patches," Ecological Entomology, vol. 29, no. 2, pp. 208-216, 2004.

[26] X. Chen, S. W. Wong, and P. A. Stansly, "Functional response of tamarixia radiata (hymenoptera: Eulophidae) to densities of its host, diaphorina citri (hemiptera: Psylloidea)," Annals of the Entomological Society of America, vol. 109, no. 3, pp. 432-437, 2016.

[27] G. Kaçar, X.-G. Wang, A. Biondi, and K. M. Daane, "Linear functional response by two pupal drosophila parasitoids foraging within single or multiple patch environments," PLoS One, vol. 12, no. 8, p. e0183525, 2017.

[28] J. Ebrahimifar, A. Jamshidnia, and H. Allahyari, "Functional response of eretmocerus delhiensis on trialeurodes vaporariorum by parasitism and host feeding," Journal of Insect Science, vol. 17, no. 2, p. 56, 2017.

[29] M. Collins, S. Ward, and A. Dixon, "Handling time and the functional response of aphelinus thomsoni, a predator and parasite of the aphid drepanosiphum platanoidis," The Journal of Animal Ecology, pp. 479-487, 1981.

[30] M. Hassell, J. Lawton, and J. Beddington, "Sigmoid functional responses by invertebrate predators and parasitoids," The Journal of Animal Ecology, pp. 249-262, 1977.

[31] V. Fernández-arhex and J. C. Corley, "The functional response of parasitoids and its implications for biological control," Biocontrol Science and Technology, vol. 13, no. 4, pp. 403-413, 2003.

[32] J. R. Beddington, "Mutual interference between parasites or predators and its effect on searching efficiency," The Journal of Animal Ecology, pp. 331-340, 1975.

[33] C. Bernstein, "Density dependence and the stability of host-parasitoid systems," Oikos, pp. 176-180, 1986.

[34] C. Free, J. Beddington, and J. Lawton, "On the inadequacy of simple models of mutual interference for parasitism and predation," The Journal of Animal Ecology, pp. 543-554, 1977.

[35] D. Rogers and M. Hassell, "General models for insect parasite and predator searching behaviour: interference," The Journal of Animal Ecology, pp. 239-253, 1974.

[36] X. Tang, L. Meng, A. Kapranas, F. Xu, I. C. Hardy, and B. Li, "Mutually beneficial host exploitation and ultra-biased sex ratios in quasisocial parasitoids," Nature Communications, vol. 5, no. 1, pp. 1-7, 2014. 
[37] R. M. May, “Limit cycles in predator-prey communities,” Science, vol. 177, no. 4052, pp. 900-902, 1972.

[38] F. Brauer, "Boundedness of solutions of predator-prey systems," Theoretical Population Biology, vol. 15, no. 2, pp. 268-273, 1979.

[39] M. P. Hassell and H. N. Comins, "Sigmoid functional responses and population stability," Theor Popul Biol, vol. 14, no. 1, pp. 62-66, 1978.

[40] A. Singh, "Population dynamics of multi-host communities attacked by a common parasitoid," bioRxiv, pp. 2021-01, 2021.

[41] A. Singh and R. M. Nisbet, "Variation in risk in single-species discrete-time models," Mathematical Biosciences and Engineering, vol. 5, pp. 859-875, 2008.

[42] B. K. Emerick and A. Singh, "The effects of host-feeding on stability of discrete-time host-parasitoid population dynamic models." Mathematical Biosciences, vol. 272, pp. 54-63, 2016.

[43] A. Singh and R. M. Nisbet, "Semi-discrete host-parasitoid models," Journal of Theoretical Biology, vol. 247, no. 4, pp. 733-742, 2007.

[44] E. Pachepsky, R. M. Nisbet, and W. W. Murdoch, "Between discrete and continuous: Consumer-resource dynamics with synchronized reproduction," Ecology, vol. 89, no. 1, pp. 280-288, 2007.

[45] B. K. Emerick and A. Singh, "Global redistribution and local migration in semi-discrete host-parasitoid population dynamic models." Mathematical Biosciences, vol. 327, p. 108409, 2020.

[46] A. Singh and B. Emerick, "Hybrid systems framework for modeling host-parasitoid population dynamics," in 2020 59th IEEE Conference on Decision and Control (CDC). IEEE, 2020, pp. 4628-4633.

[47] A. Singh, "Fluctuations in population densities inform stability mechanisms in host-parasitoid interactions," bioRxiv, 2020.

[48] J. D. Reeve, J. T. Cronin, and D. R. Strong., "Parasitoid aggregation and the stabilization of a salt marsh host- parasitoid system," Ecology, vol. 75, pp. 288-295, 1994.

[49] P. Rohani, H. C. J. Godfray, and M. P. Hassell, "Aggregation and the dynamics of host-parasitoid systems: A discrete-generation model with within-generation redistribution," The American Naturalist, vol. 144, no. 3, pp. 491-509, 1994.

[50] R. M. May, "Host-parasitoid systems in patchy environments: a phenomenological model," Journal of Animal Ecology, vol. 47, pp. 833-844, 1978.

[51] A. D. Taylor, "Heterogeneity in host-parasitoid interactions: 'aggregation of risk' and the 'cv ${ }^{2}>1$ rule.'," Trends in Ecology and Evolution, vol. 8, pp. 400-405, 1993.

[52] M. P. Hassell, R. M. May, S. W. Pacala, and P. L. Chesson., "The persistence of host-parasitoid associations in patchy environments. I. a general criterion." American Naturalist, vol. 138, pp. 568-583, 1991.

[53] S. W. Pacala and M. P. Hassell., "The persistence of host- parasitoid associations in patchy environments. II. evaluation of field data." American Naturalist, vol. 138, pp. 584-605, 1991.

[54] A. Singh, "Generalized conditions for coexistence of competing parasitoids on a shared host," bioRxiv, 2020.

[55] T. Okuyama, "Dilution effects enhance variation in parasitism risk among hosts and stabilize host-parasitoid population dynamics," Ecological Modelling, vol. 441, p. 109425, 2021.

[56] A. Oaten and W. W. Murdoch, "Functional response and stability in predator-prey systems," The American Naturalist, vol. 109, no. 967, pp. 289-298, 1975.

[57] W. S. C. Gurney and R. M. Nisbet, Ecological Dynamics. Oxford University Press, 1998.

[58] G. Ledder, Mathematics for the life sciences: calculus, modeling, probability, and dynamical systems. Springer Science \& Business Media, 2013.

[59] S. Elaydi, An Introduction to Difference Equations. Newyork: Springer, 1996. 